EPJ Web of Conferences 108, 02014 (2016)

DOI: 10.1051/epjconf/201610802014

(C) Owned by the authors, published by EDP Sciences, 2016

\title{
Numerical Simulation of the Perturbed KdVB Equation
}

\author{
Alexander Bogdanov ${ }^{1, a}$ and Vladimir Mareev ${ }^{1, b}$ \\ ${ }^{1}$ St. Petersburg State University, Universitetskii prospect 35, 198504 St.Petersburg, Russia
}

\begin{abstract}
The solution of nonintegrable nonlinear equations is very difficult even numerically and practically impossible by standard analytical techniques. New view, offered by heterogeneous computational systems, gives new possibilities, but also need novel approaches for numerical realization of pertinent algorithms. We give some examples of such analysis on the base of nonlinear wave's evolution study in multiphase media with chemical reaction.
\end{abstract}

\section{Introduction}

The development of heterogeneous computing systems opens up new possibilities for the analysis of nonlinear evolution equations. They make it possible to achieve the necessary stability and accuracy of the calculations on sufficiently coarse grids to provide the possibility of evolving up to asymptotic behavior predicted by the theory. This gives rise to two types of problems - initial distributions with large gradients, and the emergence of systematic phase shifts due to the simple numerical approximation of the derivatives. The analysis of various numerical realizations of the evolution of nonlinear waves on the example of the multi-phase environment provides practical advice on building effective approaches to their implementation.

\section{Governing Equation}

The governing equation is

$$
v_{t}+v v_{x}+\alpha v_{x x}+\beta v_{x x x}=\gamma I(v)
$$

with $\alpha$ being the measure of dissipational effects, $\beta$ being the measure of dispersion and expressed via transport coefficients and relaxation times, $\gamma$ is the measure of the interphase interactions and expressed via integral brackets and relaxational times, and $I(v)$ is the integral operator.

\section{Finite Difference Scheme for KdVB Equation}

In integrating the KdVB equation, instead of the original equation (1) we use its equivalent, written in divergence form:

$$
v_{t}+0.5\left(v^{2}\right)_{x}+\alpha v_{x x}+\beta v_{x x x}=\gamma I(v) .
$$

\footnotetext{
a e-mail: a.v.bogdanov@spbu.ru

be-mail: map@csa.ru
} 
The solution of (2) in the half-plane $t \geq 0$ is sought for the initial distribution $v(x, 0)=f(x)$ at $x \in(-\infty, \infty)$. The boundary conditions are the Dirichlet conditions: $v(-\infty, t)=a, v(\infty, t)=b$.

Basically, numerical simulation of the equation (2) was carried out using a two-step explicit finitedifference McCormack scheme [1] [2] with flux-corrected technique (FCT) [3]. Also other numerical approaches are available, in particular the Zalesak generalization for hyperbolic systems [4].

The calculation is performed in the computing domain $\left[x_{\min }, x_{\max }\right] \times[0, T]$. The finite-difference grid in time $t$ and space $x$ coordinates is taken uniform:

$$
v_{j}^{n}=v\left(x_{j}, t^{n}\right), \quad x_{j}=j \Delta x, j \in[1, M], x_{1}=x_{\min }, x_{M}=x_{\max }, t^{n}=n \Delta t, n=0,1, \ldots,
$$

with $\Delta x$ being the step on spatial coordinate; $\Delta t$ being the time step. The boundary conditions: $v\left(x_{\min }, t\right)=a, v\left(x_{\max }, t\right)=b$.

The McCormack difference scheme for (2) has the form $\left(r=\alpha \Delta t / \Delta x^{2}, s=\beta \Delta t /\left(2 \Delta x^{3}\right)\right)$ :

Step 1. Predictor.

$$
v_{j}^{*}=v_{j}^{n}-\frac{\Delta t}{2 \Delta x}\left[\left(v_{j+1}^{n}\right)^{2}-\left(v_{j}^{n}\right)^{2}\right]-r\left(v_{j+1}^{n}-2 v_{j}^{n}+v_{j-1}^{n}\right)-s\left(v_{j+2}^{n}-2 v_{j+1}^{n}+2 v_{j-1}^{n}+v_{j-2}^{n}\right)+\gamma \Delta t I_{j}^{n}
$$

Step 2. Corrector.

$$
\bar{v}_{j}=\frac{v_{j}^{n}+v_{j}^{*}}{2}-\frac{\Delta t}{4 \Delta x}\left[\left(v_{j}^{*}\right)^{2}-\left(v_{j-1}^{*}\right)^{2}\right]-\frac{r}{2}\left(v_{j+1}^{*}-2 v_{j}^{*}+v_{j-1}^{*}\right)-\frac{s}{2}\left(v_{j+2}^{*}-2 v_{j+1}^{*}+2 v_{j-1}^{*}+v_{j-2}^{*}\right)+\gamma \frac{\Delta t}{2} I_{j}^{*}
$$

The approximation of the transport term in the predictor step for odd time steps is performed by forward differences and for the even time steps by backward differences. Thus, the phase error is reduced in the calculations and wave fronts move in correct directions.

It is known that the McCormack scheme, while improving the order of the approximation as compared with the monotone schemes, generates nonphysical oscillations in the vicinity of strong jumps.

The FCT method according to [2] includes the following stages:

1. The calculation of the diffusive fluxes: $v_{j+1 / 2}^{d}=v_{j+1 / 2}\left(v_{j+1}^{n}-v_{j}^{n}\right)$.

2. The calculation of the antidiffusive fluxes: $v_{j+1 / 2}^{a d}=\mu_{j+1 / 2}\left(\bar{v}_{j+1}-\bar{v}_{j}\right)$.

The coefficients $v_{j+1 / 2}$ and $\mu_{j+1 / 2}$ are specified as

$$
v_{j+1 / 2}=\frac{1}{6}+\frac{1}{3} C_{j+1 / 2}^{2}, \quad \mu_{j+1 / 2}=\frac{1}{6}-\frac{1}{3} C_{j+1 / 2}^{2}, \quad C_{j+1 / 2}=\frac{v_{j}^{n}+v_{j+1}^{n}}{2} \frac{\Delta t}{\Delta x} .
$$

After the correction step we get the adjusted value $v_{j}^{\text {corr }}$. The final decision on the layer $n+1$ has the form:

$$
v_{j}^{n+1}=\bar{v}_{j}+v_{j+1 / 2}^{d}-v_{j-1 / 2}^{d}-v_{j}^{\text {corr }}+v_{j-1}^{\text {corr }} .
$$

\section{Initial Value Problems}

In calculations as the starting initial profiles two distributions are used:

1. "Shock wave" (jump): $\left.v\left(x \geq x_{0}, 0\right)=u_{1}\right) ; v\left(x<x_{0}, 0\right)=u_{2}$. The speed of the jump shift: $U=\left(u_{1}+u_{2}\right) / 2=$ const.

2. Soliton. The known stationary solution of the KdV equation:

$$
v(x, 0)=f(x)=\varepsilon \cosh ^{-2} x / \delta, \quad \delta=\sqrt{12 \beta / \varepsilon} .
$$


3. The rectangular profile. $v(x, 0)=h \times w$, at $x \in[-w / 2, w / 2]$. The choice between $h$ and $w$ is dictated by the conservation of "mass" for source profile:

$$
\int_{-\infty}^{\infty} f(x) d x=\int_{-\infty}^{\infty} \varepsilon \cosh ^{-2} x / \delta d x=4 \sqrt{3 \varepsilon \delta}=h \times w
$$

In our case (4), the expression (5) has the form: $h \times w=12$. To maintain this equality in calculation, the specific value of $w$ should also be associated with a step for $x$-coordinate.

\subsection{Numerical Tests}

To test the finite-difference scheme described in section 3, we consider special cases of the original equation (1): transport, Burgers equation, and $\mathrm{KdV}$ equation. The basic properties of the difference schemes (stability, order of approximation, the modified equation, etc.) are described in [1], [2].

\subsection{Convection}

In the case of pure transport, the equation (2) has the following form:

$$
v_{t}+0.5\left(v^{2}\right)_{x}=0
$$

The finite-difference scheme for transport was traditionally tested by "shock wave". The jump has a unit amplitude: $u_{1}=1, u_{2}=0, x_{0}=0$. The average transfer rate $U$ is then equal to $1 / 2$.

The linearized form of the equation (6) is: $v_{t}+U v_{x}=0$.

On fig. 1 the results of the numerical calculations for time $=125, C=0.25$ and $x=U t=62.5$ are shown. Comparison of the results of numerical calculations with the analytical solution for the distribution of "shock front" shows excellent agreement.

For the linear case of (6), with the procedure of flux correction FCT, there is a known stability condition: $C \leq 1 / 2$, where $C=$ $U \Delta t / \Delta x$ is the Courant number and $\Delta t, \Delta x$ are the steps in time and coordinates. For the nonlinear case the stability condition is transformed to

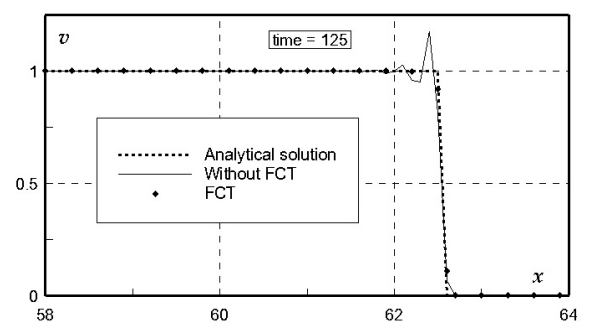

Figure 1. Comparison of the results for the distribution of "shock front"

$$
\left.\mid \max _{\forall j, n} v_{j}^{n} \Delta t / \Delta x\right) \mid \leq 1 / 2
$$

From (5) the influence of the dispersion error is clearly visible depending on the Courant number. Without flow correction algorithm (FCT), we can not get the correct numerical solution.

Calculations show that the limiting step $\Delta t$ in time for convection at $\Delta x=0.1$ is $0.071(C=0.355)$. 


\subsection{Burgers Equation}

At $\beta=\gamma=0$, the equation (2) is converted to the Burgers equation:

$$
v_{t}+v v_{x}+\alpha v_{x x}=0 .
$$

For this equation (7) there is a lot of exact solutions [5]. In our case, we use as the testing scheme the stationary analytical solution:

$$
u=\frac{u_{2}-u_{1}}{1+\exp \left(-\frac{u_{2}-u_{1}}{2 \alpha} \xi\right)}, \quad \xi=x-U t .
$$

As in the case of convection 4.2 for the initial distribution, we take a unit jump: $u_{1}=$ $1, u_{2}=0, x_{0}=0$. In fig. 2 the analytic solution is compared with the evolution of a unit jump and the jump without the influence of the viscosity.

The dominant dissipative effect is the physical diffusion, which suppresses the numerical dispersion error.

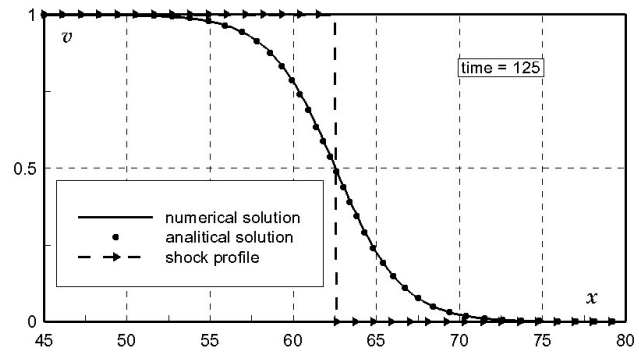

Figure 2. Comparison of numerical and analytical solutions for Burgers equation

\subsection{KdV Equation}

When $\alpha=\gamma=0$ the equation (2) is converted to the KdV equation:

$$
v_{t}+0.5\left(v^{2}\right)_{x}+\beta v_{x x x}=0 .
$$

Equation (8) has no dissipative terms, and therefore the numerical results are very sensitive to errors of approximation, especially to phase errors. And not only terms with lower derivatives have the effect of the approximation errors but also terms with derivatives of higher orders.

We are faced with the fact that the direct application of the described above finite-difference method leads to erroneous results. This is due to the fact that the correction procedure itself has a grid diffusion. The results taking into account the correction procedure, as expected, are quite satisfactory.

Therefore, a simple reduction in the time step can significantly affect the resulting solution. We calculated the distributions for different time steps compared with the analytical solution (4). The speed of the soliton propagation $U=\varepsilon / 3$ for stationary solutions $(\varepsilon=12)$ is $4\left(r=\beta \Delta t /\left(2 \Delta x^{3}\right)\right.$. The speed of motion of the soliton in case 2 of fig. 3 is about 3.97 , with a relative error equal to $0.64 \%$, which is quite a decent result. The relative error of the estimated amplitude of the soliton is $0.58 \%$.

We considered other schemes for equation (8), in particular three-layer explicit schemes

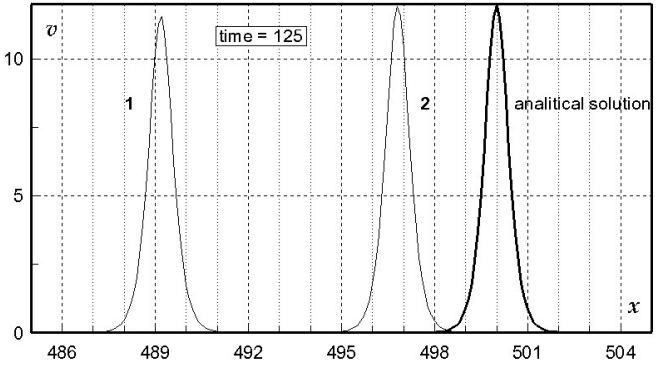

Figure 3. Comparison of solutions for different time steps: $1-r=6.25 \cdot 10^{-3} ; 2-r=6.25 \cdot 10^{-4}$ with different orders of approximation to the convective term. All of them show poor results. 
When considering the rectangular profile without dissipative effects we observed numerical dispersion, which is well illustrated in fig. 4.

One can achieve acceptable results of calculation for the $\mathrm{KdV}$ equation (8), if we will find the reduced diffusion and antidiffusive coefficients (3) for fluxes correction procedure. On fig. 5 we show one possible choice of the coefficients $v_{j+1 / 2}=0.000111 ; \mu_{j+1 / 2}=0.00011$. The initial distribution for fig. 4 and fig. 5 is the following: $h \times w=4.15 \times 2.6, r=6.25 \cdot 10^{-4}$.

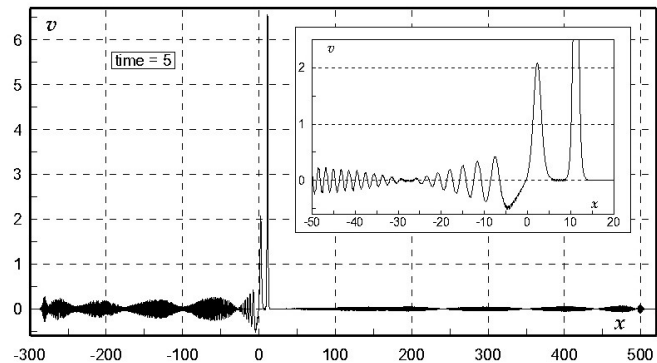

Figure 4. McCormack scheme for (8): the effects of numerical dispersion, $h \times w=4.15 \times 2.6, r=6.25 \cdot 10^{-4}$

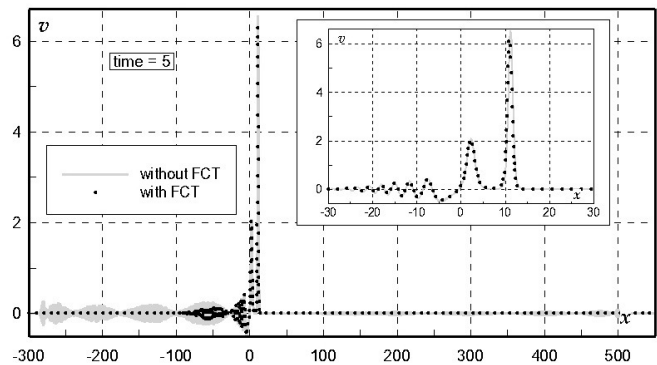

Figure 5. Comparison of the results for $\mathrm{KdV}$ equation with and without correction, for coefficients choice $v_{j+1 / 2}=0.000111 ; \mu_{j+1 / 2}=0.00011$

\section{KdVB Equation Modeling}

For the KdVB equation (1) the integral operator is: $I(v)=-\int_{0}^{t} G(t, \tau) v_{\tau} d \tau, G(t, \tau)=e^{-k(t-\tau)}, k>0$.
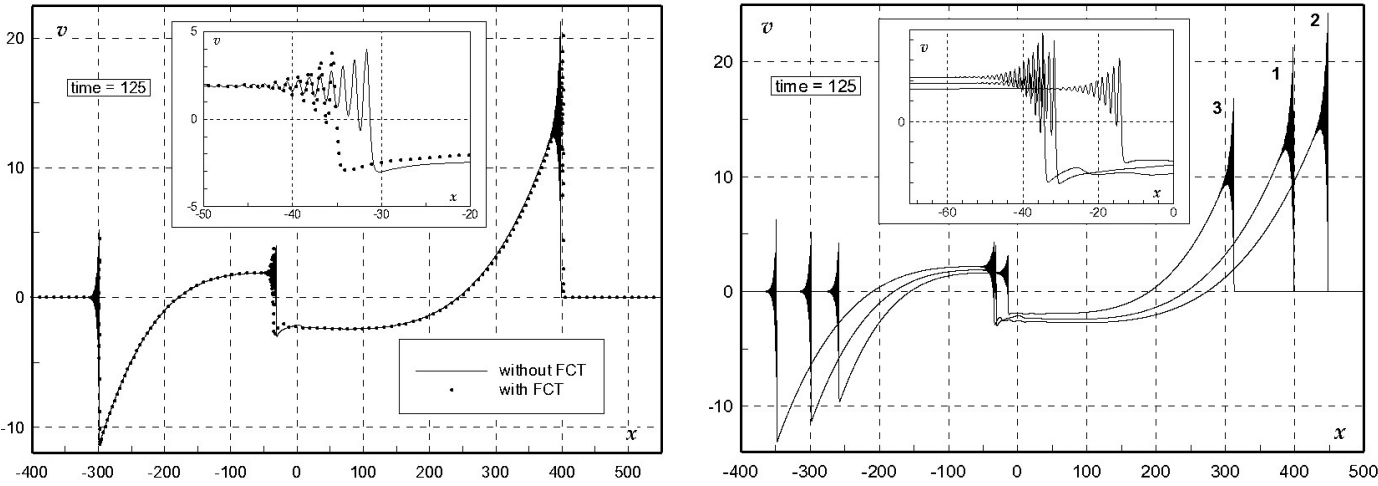

Figure 6. $h \times w=4.15 \times 2.9 ; r=6.25 \cdot 10^{-4} ; \alpha=-0.05, \quad$ Figure 7. $1-h \times w=4.15 \times 2.9 ; 2-h \times w=11 \times 1.1$; $\beta=\gamma=k=0.1 ; v_{j+1 / 2}=0.000111, \mu_{j+1 / 2}=0.00011 \quad 3$-soliton: $\varepsilon=12, \delta=0.5 ; r=6.25 \cdot 10^{-4} ; \alpha=-0.05$, $\beta=\gamma=k=0.1$

When considering the complete equation (1) the requirements for the selection of the difference scheme are reduced. The nonzero right side of the equation acts as a source. The effect of the flow correction is not as significant as in the absence of diffusion factors, even at low values of the coefficient. This is well illustrated in fig. 6, which deals with the initial "jump" rectangular profile 4.2. Both the form and the amplitude of the velocity distributions are practically identical. 
In the following fig. 7 we compared the results without correction procedure for the same parameters as in fig. 6 for the rectangular profile 4.2 and soliton like initial distribution (3). The only criterion for comparison is the initial "weight" of the distribution, which is equal to about 12. Due to pumping of the mass from RHS the acceleration occurs in the initial distribution and the increase in amplitude. The very form of the distribution of the perturbation is the same for all source profiles.

\section{Conclusion}

Four fairly obvious brief conclusions follow from the above results.

1. In the consideration of KdVB (1), in our opinion it does not make much sense to try to create a universal finite-difference scheme. Versatility in this case, means the use of the average characteristics of the error of approximation and generation in certain cases, numerical dissipative and dispersion terms. That is extremely undesirable.

2. The differential approximation for the explicit finite-difference McCormack scheme even for the Burgers equation (3) in the linear approximation is very cumbersome. In this case, it is not enough to consider only the principal terms of the differential approach, taking into account the order of approximation of the difference scheme. Numerical experiments have shown a great influence on the result coming from the expansion terms originating in the higher derivatives. These errors cause significant phase errors with the change of the time step.

3 . It is confirmed that the procedure for flux correcting is useful mainly when we do not have an explicit diffusion term $(\alpha=0)$. Embodiments of the FCT method, as in the case of the Zalesak approach [4] did not give any qualitative advantages over the old proven method [4]. Trying to complete the calculation of the equation KdVB by other algorithms also do not give proper effect. Rather, it led to poor results. Using implicit difference schemes for obvious reasons it is not possible.

4. As used in this paper, the method of calculation is quite clear and straightforward to implement with sufficient accuracy. Later, in our opinion, it is necessary to analyze more thoroughly the approximation error for decisive conclusions. Some ideas to turn to 3D computations effectively were discussed in our presentation [6].

\section{Acknowledgements}

The research was carried out using computational resources of Resource Center Computer Center of SaintPetersburg State University and supported by Russian Foundation for Basic Research (project N 13-07-00747) and St. Petersburg State University (projects N 9.38.674.2013, 0.37.155.2014).

\section{References}

[1] Fletcher R.H., Tannehill J.C., and Anderson D., Computational Fluid Mechanics and Heat Transfer, Third Edition (Series in Computational and Physical Processes in Mechanics and Thermal Sciences, CRC Press, 2013) 774 p.

[2] Fletcher C.A.J., Computational Techniques for Fluid Dynamics 2: Specific Techniques for Different Flow Categories, (Springer-Verlag, 1998) 496 p.

[3] Boris J. P. and Book D. L., J. Comput. Phys. 11, 38-69 (1973)

[4] Zalesak S.T., J. Comput. Phys. 31, 335-362 (1979)

[5] Benton E. R. and Platzman G. W., Quart. Appl. Math. 30, 195-212 (1972)

[6] Bogdanov A., Stankova E., and Mareev V., High performance algorithms for multiphase and multicomponent media, 14th Ship stability workshop, UTMSPACE, Malaysia, pp. 242-245 (2014) 